\title{
LA EXPLOTACIÓN DE RECURSOS EN LOS MÁRGENES DEL VALLE DEL NILO DURANTE EL REINO MEDIO: LA DINASTÍ́A XII
}

\author{
The Exploitation of Resoures in the Nile Valley Margins during the Middle Kingdom: \\ The Twelfth Dynasty
}

\author{
Javier GONZÁLEZ-TABLAS NIETO* \\ Universidad de Salamanca \\ E-mail: jgtablas@usal.es
}

Fecha de recepción: 20-01-2010

Fecha de aceptación: 29-01-2010

RESUMEN: Los márgenes del Valle del Nilo siempre estuvieron en el punto de mira de los faraones, debido a la riqueza y variedad de productos que aportaban, desde los importantes recursos minerales obtenidos en el desierto oriental o el Sinaí, hasta los exotica y aromata que llegaban al país a través de Nubia . En los periodos de mayor debilidad de la monarquía egipcia, estas zonas solían caer en desuso, como ocurrió durante el Primer Periodo Intermedio. Pero con la reunificación del país en el Reino Medio, la actividad egipcia en el exterior conoció un nuevo impulso, documentándose expediciones mineras en casi todos los yacimientos conocidos, así como la presencia egipcia en las zonas limítrofes, como Kerma, Siria-Palestina o Punt. La naturaleza de las relaciones entre los egipcios y los habitantes nativos de estas regiones puede vislumbrarse a través del estudio de las inscripciones y vestigios arqueológicos que dejaron tras de sí.

Palabras Clave:Egipto faraónico, Reino Medio, dinastía XII, explotación de recursos, minería, comercio.

ABSTRACT: The Nile Valley margins were always one of the aims of the pharaohs, due to the abundance and variety of products they had, ranging from the important mineral resources obtained in the Eastern desert or the Sinai, to the exotica and aromata that came to the country through Nubia. In the times when the egyptian monarchy was weak, these 
regions were less used, like during the First Intermediate Period. But, after Middle Kingdom reunification, egyptian activity outside the country saw a new boost, quarrying expeditions being documented in almost every known site, and so the egyptian presence in the neighboring regions, such as Kerma, Syria-Palestine or Punt. The nature of the relationship between the egyptians and natives from these regions can be glimpsed through the study of the inscriptions and archaeological remains these people left behind them.

Keywords: Pharaonic Egypt, Middle Kingdom, Twelfth Dynasty, Resources exploitation, Quarrying, Trade.

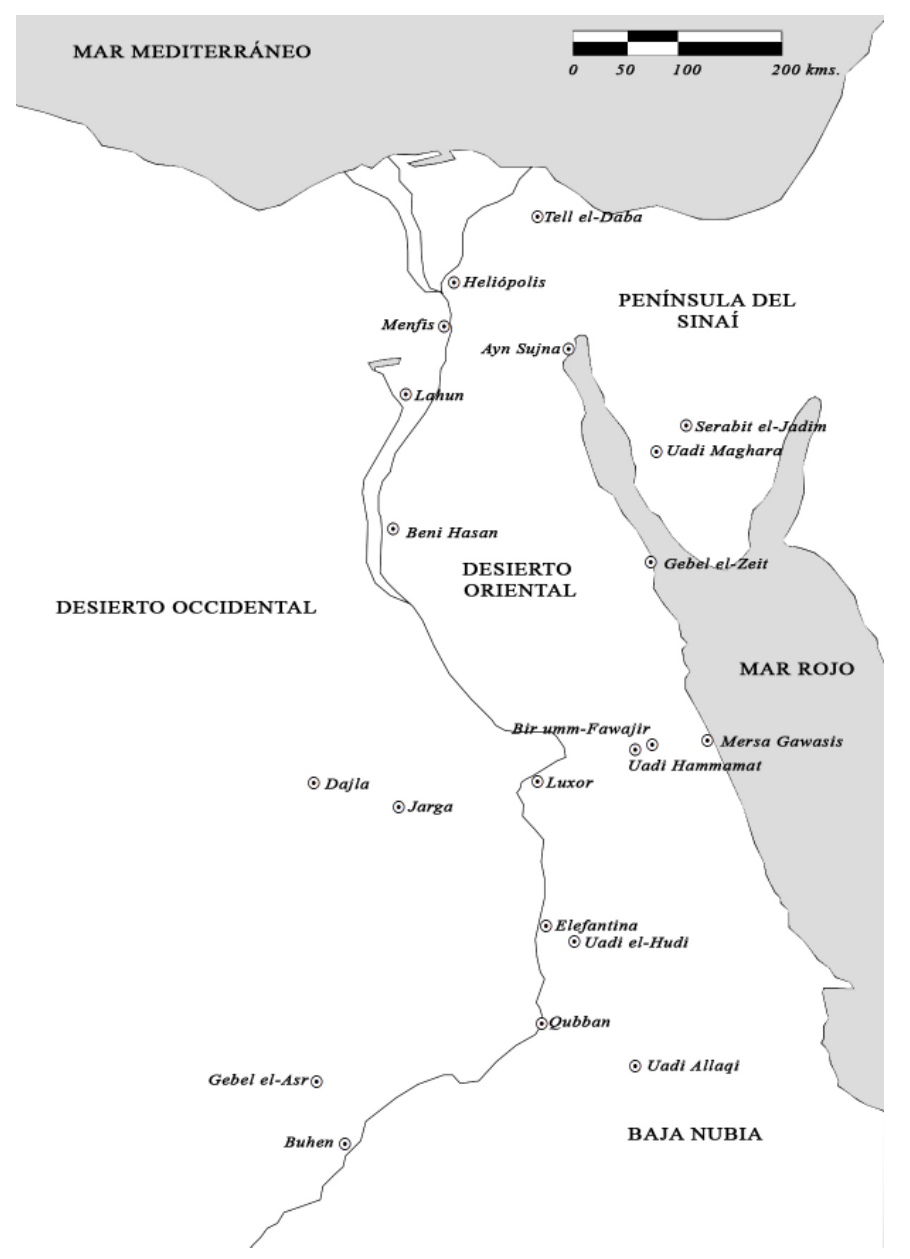

Fig.1 Mapa de los lugares mencionados en el texto 
Hay ciertas frases que marcan la visión de una civilización. Este es el caso de Egipto y la célebre definición del griego heródoto como don del Nilo, tan conocida como cierta, pero que puede llevar a malinterpretaciones sobre la relación de los egipcios con su entorno. Si bien es cierto que la crecida anual del Nilo, que anegaba con un limo fértil las orillas del río, regía la vida egipcia, , no todo lo que la sociedad egipcia necesitaba se podía encontrar en el valle.

El verde valle se encuentra rodeado de áridos desiertos, y regiones que bien por su orografía o su clima resultaban de difícil habitación. Al este, el desierto oriental abarca todo el territorio desde el Valle hasta el Mar Rojo. $\mathrm{Al}$ oeste, el desierto libio, salpicado de oasis y rutas de caravanas. A sur, las cataratas del río Nilo que dificultaban enormemente su navegación y que se erigían en fronteras naturales. Y al norte, el mar Mediterráneo y las otras grandes civilizaciones del Oriente Próximo.

En estas cirunstancias geográficas, la relación del Egipto faraónico con su entorno resultaba fundamental en el desarrollo de su cultura. Desde muy antiguo, Egipto explotó las zonas limítrofes, que ofrecían muy variados recursos, desde minerales, maderas, aromata y exotica hasta mano de obra en forma de prisioneros. La intensidad de la explotación de estas regiones dependió en gran medida de la estabilidad interna del país. Así, durante el Reino Antiguo, los desiertos fueron explotados en busca de minerales, y se controló mediante la fuerza militar la zona de la Baja Nubia. Durante el Primer Periodo Intermedio, este control disminuyó notablemente, volviendo a repuntar durante la dinastía XI tras la reunificación.

El periodo objeto de esta investigación, la dinastía XII, es uno de los momentos de mayor estabilidad interna en Egipto ${ }^{1}$. Con unas fronteras exteriores bien consolidadas, los faraones pudieron concentrarse en el envío de expediciones, en unos casos mineras, en otros comerciales, así como diplomáticas, a las zonas limítrofes. Estas expediciones acostumbraban a erigir monumentos relatando su viaje, organización, fecha, etc., aunque la información aportada por estos documentos es muy variable, dependiendo de su estado de conservación, o incluso, de no haber sido aún descubierto.

La investigación de la explotación de estas zonas se realiza mediante el examen estos materiales, especialmente las inscripciones relacionadas con las expediciones. Estas inscripciones, muy variables en cuanto a su cantidad, contenido, y periodo abarcado, son una fuante de información única que permite, en muchos casos, una reconstrucción más o menos precisa de la logística de estas expediciones, completada en la medida de lo posible por los hallazgos arqueológicos.

Las razones que tuvo la administración egipcia para el envío de estas expediciones variaba enormemente según la zona que se estudie. 


\section{NuBIA}

Al hablar de la zona conocida como la Baja Nubia, las relaciones de Egipto con esta región (wawat en egipcio antiguo) se remontan a los comienzos del Estado faraónico ${ }^{2}$. Desde muy temprano, los egipcios fueron conscientes de la necesidad de crear un espacio controlado y protegido al sur de Elefantina, la ciudad más meridional del país. En el Reino Antiguo, esta región fue conquistada y militarizada, quedando fuera del control egipcio en los momentos de conflicto interno en el país. Esta tónica se mantendría a lo largo de toda la historia egipcia, coincidiendo habitualmente los momentos de fortaleza de la monarquía egipcia con un férreo control de Nubia, que recuperaba su independencia durante los denominados Periodos Intermedios.

En el periodo objeto de la presente investigación, la dinastía XII, se erigieron en esta región una serie de fortalezas militares que creaban una red de defensas contra una de las culturas que los egipcios del momento consideraban como más amenazadora: Kerma, en el actual Sudán ${ }^{3}$. Por tanto, los materiales conservados de esta región son muy abundantes, pero se circunscriben generalmente al ámbito militar. Así, son frecuentes los graffiti de soldados, guardias o personal civil asignado a estas fortalezas. Uno de los problemas que aparecen a la hora de estudiar estas inscripciones es su datación, frecuentemente muy problemática. Esta datación, que habitualmente se reduce a abanicos temporales amplios, se hace aplicando criterios epigráficos, estilísticos o lingüísticos, con los problemas que esto conlleva ${ }^{4}$. El otro gran problema al que se enfrenta el investigador de esta región es al mismo tiempo la causa de la mayor parte del conocimiento que de ella se tienen: la construcción de la gran presa de Asuán, y la campaña de salvamento organizada por la UNESCO en los años sesenta del pasado siglo. Esta campaña acometió la excavación y documentación de numeros yacimientos de la zona, que quedarían sumergidos bajo las aguas del lago Nasser. Es frecuente, por tanto, que toda la investigación deba hacerse tomando como base los informes de estas excavaciones, ya que el examen in situ de los monumentos en cuestión es, por el momento, imposible.

Pero Nubia no era solamente una fuente de amenazas y prisioneros para los egipcios. También era muy conocida por sus materias primas, algunas de ellas muy empleadas en el arte egipcio como el oro o la amatista. Poco se conoce sobre las explotaciones auríferas de la región en esta época, aunque varias zonas dedicadas a estos trabajos han sido localizadas ${ }^{5}$. El estudio de estos hábitats mineros es mucho más complicado que las fortalezas o los templos, ya que habitualmente las construcciones estaban realizadas en materiales perecederos, como la madera. La zona más conocida y citada como fuente del oro es el Uadi Allqi, para cuya protección se cree que se construyó la fortaleza de Qubban ${ }^{6}$. 
El caso de la amatista es diferente. Esta piedra semipreciosa se extraía de Uadi el-Hudi, una zona situada a unos 20 kms. al sudeste de Asuán7. Esta región cuenta con numerosos restos arqueológicos diseminados en varios yacimientos. Los restos pertenecientes a finales del Reino Medio se concentran en torno a varios elementos principaesl, que son un hábitat fortificado en una colina adyacente a una mina de amatista, y una pequeña fortaleza. Pero el rasgo característico de Uadi el-Hudi son las estelas 8 . Estas inscripciones permiten reconstruir en cierta medida las expediciones mineras enviadas a la zona, y se puede establecer como mínimo una línea cronológica en el envío de las mismas. Aunque hay que ser conscientes en todo momento de la posibilidad de que existan aún estelas por descubrir o expediciones que no dejasen restos escritos, se sabe gracias a estas inscripciones que el Reino Medio fue una de las épocas de mayor intensidad en la explotación de la amatista en la zona.

Asimismo, Nubia se consideraba una fuente de propaganda y reputación por los faraones. El caso de Senusert III es un gran ejemplo. Este faraón desarrolló una intensa actividad militar en la baja Nubia, estableciendo finalmente la frontera de Egipto en la Tercera Catarata, la más meridional hasta la fecha. Esta frontera fue conmemorada por dos estelas gemelas en las fortalezas de Semna y Kumma, que controlaban el paso del Nilo en esta Tercera Catarata. En estas estelas el faraón se vanagloria de su victoria sobre los nubios, a los que tilda de "cobardes" y "miserables", una terminología muy habitual en estos textos.

Pero pese a este tipo de lenguaje, debemos ser muy cautos a la hora de considerar a esta civilización nubia. $Y$ es que si analizamos la zona sobre un mapa, resulta un tanto sorprendente la cantidad de recursos tanto materiales como humanos que se emplearon a lo largo de toda la dinastía XII para construir y guarnecer estas fortalezas, si hablamos de un pueblo "miserable" y "cobarde" " La arqueología ayuda a aclarar un poco esta situación: las recientes excavaciones de un equipo suizo dirigido por Ch. Bonnet en Kerma, al sur de la Tercera Catarata (actual territorio de Sudán), han revelado los restos de un importante centro urbano con una habitación ininterrumpida desde épocas que se remontan al comienzo de la civilización egipcia. Esto permite saber, por tanto, que los egipcios siempre tuvieron presentes a estos vecinos del sur, que al igual que los faraones, gozaron de mayor o menor poder según las circunstancias.

Se puede observar, por tanto, que en el caso de Nubia, la presencia de una población indígena marca la tónica de la explotación de la zona, supeditada a la capacidad por parte de Egipto de proporcionar protección a las expediciones. 


\section{EL DESIERTO ORIENTAL}

El caso del desierto que se extiende desde el Valle hasta el Mar Rojo es distinto al de Nubia, principalmente debido a la ausencia de una población indígena fuerte en la región. La explotación que los egipcios hicieron de esta zona se centró eminentemente en la salida que proporcionaba al Mar Rojo. Si bien es cierto que hay ciertos yacimientos que eran explotados por su propio valor material, lo habitual es que los yacimientos importantes se localicen en la costa oriental. Los yacimientos más importantes para esta investigación gracias al material arqueológico y epigráfico que aportan son, de norte a sur, Ayn Sujna, Gebel el-Zeit, Mersa Gawasis, y, en el interior del desierto, el Uadi Hammamat.

Ayn Sujna, una región minera que conecta la región de Menfis con el golfo de Suez, fue el objetivo de varias expediciones en el periodo que nos atañe ${ }^{10}$. Uno de los objetivos más claros, y confirmado tanto por las inscripciones como por los vestigios arqueológicos, es el mineral de cobre. La explotación de este material se realizaba in situ, y se han excavado varias galerías mineras. Pero otro objetivo, menos claro, parece haber sido la ruta marítima hacia el Sinaí. Esta región, que analizaremos más adelante, era rica en turquesa, además de cobre. Hay una inscripción en Ayn Sujna que se puede conectar directamente con una expedición enviada al Sinaí en el año 2 de Amenemhat III ${ }^{11}$, lo que ha planteado la posibilidad de que estas expediciones recorriesen por tierra el camino hasta Ayn Sujna, y tomasen un barco hasta la zona sedimentaria de la desemocadura del Uadi Baba, desde donde podrían haber tomado el camino tanto a Uadi Maghara como a Serabit elJadim.

Gebel el-Zeit es un yacimiento compuesto por un pequeño santuario y minas de galena. Las excavaciones del IFAO han revelado varias inscripciones del Reino Medio en un depósito votivo en el santuario, además de numerosos escarabeos ${ }^{12}$.

Mersa Gawasis es el único yacimiento que ha sido catalogado de puerto con cierta seguridad en el Reino Medio ${ }^{13}$. Se han descubierto numerosos restos de anclas, así como varias estelas de la época que vinculan este puerto con expediciones marítimas a Punt, una región cuya identificación exacta sigue siendo dudosa, pero cuyo comercio resultaba muy valioso para los egip$\operatorname{cios}^{14}$.

El Uadi Hammamat merece unas líneas aparte. Se trata de una región a medio camino entre Coptos y Mersa Gawasis, y es una de las canteras más importantes en el Reino Medio. De sus sinuosos valles se extraía la piedra bejen, conocida hoy en día como grauvaca ${ }^{15}$, y que se trataba de una piedra muy valiosa para los faraones, empleada especialmente en sarcófagos y estatuas. Hay que resaltar que esta es la única cantera del todo el país de la que 
se extraía esta piedra, por lo que no sorprende la intensa actividad que hay documentada en esta zona. Son muy numerosas las inscripciones rupestres de esta época que se han encontrado ${ }^{16}$. Estas inscripciones, grabadas en la roca del uadi, relatan las expediciones egipcias y los problemas que encontraban, además de la estructura administrativa de las mismas. Un ejemplo puede verse en la inscripción WH 19, correspondiente al año 19 de Amenemhat III:

Año 19 bajo la majestad del Rey Dual Nymaatre, el bijo de Re Amenembat, iQue le sean dadas vida, estabilidady dominio como a Re!

Su majestad decretó que se le trajeran monumentos de esta montaña noble al oeste del valle. Asípues se deslizó la piedra de esta montaña occidental como se hacía antes. Entonces, esta piedra sobre el se deterioró hasta romperse. Nunca se encontró una que llegase bien abajo.

Entonces dijo el controlador de los trabajos, el heraldo del tribunal Mery: ¡Vamos! que se construya una rampa para deslizar la piedra. Entonces se construyó esta rampa, y se hocieron deslizar los monumentos como él habia dicho, lo que nunca antes se habia hecho. Así, él presentó diez. nobles estatuas de cinco (codos).

Su expedición de canteros: 20; de mineros, 30; de remeros 30. Conjunto de la expedición, $2000^{17}$.

En ella, el encargado de la misma, el controlador de los trabajos Mery, relata cómo se fracturaron las piedras al deslizarlas montaña abajo como era costumbre en este tipo de operaciones. Para solucionar este problema, ordenó construir una rampa para deslizar las piedras, consiguiendo llevar de vuelta al valle 10 estatuas de cinco codos de altura. A continuación describe los obreros que componían la expedición. Esta inscripción muestra varias cosas muy interesantes para nuestra investigación. En primer lugar, muestra los objetivos y problemas de la expedición; en segundo lugar, la iniciativa y capacidad logística que tenían los líderes expedicionarios en Egipto, y, por último, la composición de los obreros que acudieron allí.

Aunque no todo es tan fácil como pueda parecer por inscripciones como ésta. En primer lugar, las inscripciones no son fáciles de leer ni de traducir, debido a que no son escritas por artistas de la corte, como suele pasar con los monumentos como estelas o tumbas, sino por los propios expedicionarios. Esto hace que posean rasgos lingüísticos propios y grafías particulares. A esto debemos sumar el hecho de que este tipo de inscripciones tan detalladas no son lo normal, y otras inscripciones que ofrecen listas de trabajadores dan cifras muy dispares. 
Además de esta aportación de documentos, el Uadi Hammamat estaba en la ruta hacia una zona de explotación aurífera, Bir Umm Fauajir. Esta zona presenta restos de explotación que datan de época faraónica, pero el momento de mayor actividad se da en época romana ${ }^{18}$.

\section{EL SINAÍ}

La península del Sinaí es una zona muy rica en recursos minerales que ha sido explotada desde el Reino Antiguo hasta la actualidad. En la época que nos ocupa, esta explotación se centraba fundamentalemente en el cobre y la turquesa. Es significativo que el cobre sólo se menciona una vez en las inscripciones, siendo la turquesa el objetivo declarado hasta la saciedad en las inscripciones halladas, precisamente, en el santuario de Hathor, "Señora de la Turquesa". Pero esta evidencia textual se contradice con los restos arqueológicos de la zona, que documentan una intensa actividad relacionada con el cobre.

Las zonas de explotación son principalmente dos, Uadi Maghara ${ }^{19} \mathrm{y}$ Serabit el-Jadim ${ }^{20}$. La primera es una zona de vadis en la que se han encontrado restos de hábitats mineros y de explotaciones, así como algunas inscripciones. Pero la zona más prolífica tanto en inscripciones como en restos arqueológicos es la meseta de Serabit el-Jadim y los uadis que la rodean. En esta meseta se encuetra el santuario de Hathor, que fue construido y ampliado a lo largo de la dinastía XII, y sería remodelado completamente durante el Reino Nuevo.

En este santuario, los expedicionarios erigieron estelas relatando sus objetivos, equipos y resultados. Estas inscripciones se colocan siguiendo un esquema no lineal pero sí siguiendo un cierto orden, generalmente asociado a las nuevas obras en el recinto. Durante el reinado de Amenemhat III, se documentan al menos 28 expediciones en un periodo de unos 45 años, el tiempo de duración de dicho reinado. Esto da una idea de la importancia de esta región y su explotación.

Las inscripciones y los restos arqueológicos del Sinaí han puesto de relieve otras particularidades. En primer lugar, resulta muy significativa la presencia, bastante abundante, de intérpretes y asiáticos en las inscripciones. Esto se explica desde la necesidad de una interacción pacífica con los habitantes de la zona. Si bien en las expediciones se documenta la presencia de militares, su número es muy inferior a las que aparecen, por ejemplo, en las de Uadi el-Hudi. Uno de estos asiáticos, el hermano del mandatario de Retyenu Jebeded, aparece en varias expediciones diferentes, incluso representado en varias estelas montando en un burro con dos siervos. A estas representaciones hay que sumar una peculiaridad de la zona, las denominadas inscrip- 
ciones protosinaíticas ${ }^{21}$. Aún bastante desconocidas, se trata de inscripciones posiblemente realizadas por semitas que habían entrado en contacto con Egipto. Aunque no son exclusivas de esta zona, sí que es su principal lugar de aparición, y se ha especulado con la posiblidad de que fuesen inscritas por estos asiáticos que participaban como guías o interpretes en las expediciones egipcias.

\section{ASIA}

Lo que hemos visto sobre el Sinaí permite enlazar directamente con el tema de los contactos entre Egipto y Asia en el Reino Medio. Estos contactos parecen haberse producido eminentemente de forma pacífica y restringidos al ámbito comercial, y son casi inexistentes los testimonios de campañas militares. Tan sólo la estela de Jusobek relata una campaña militar en el reinado de Senusert III contra los asiáticos ${ }^{22}$, aunque es difícil precisar el significado exacto del término "asiático" en egipcio ${ }^{23}$.

Se han hallado restos egipcios en la zona de Siria-Palestina, y testimonios literarios como la historia de Sinuhé demuestran un cierto conocimiento por parte de los egipcios de sus vecinos del norte y sus costumbres. Además, los textos oficiales, como los anales de Amenemhat II de Menfis ${ }^{24}$, confirman contactos diplomáticos con representantes de pueblos de Asia, así como los textos recientemente reestudiados por J. P. Allen de la mastaba de Jnumhetep en Dashur ${ }^{25}$.

En cualquier caso, resulta muy complicado con los datos de que disponemos el elaborar un esquema preciso de las relaciones entre Egipto y Asia en este momento.

\section{EL DESIERTO OCCIDENTAL}

A diferencia de lo que sucedía con el desierto oriental, el desierto occidental sufrió una explotación mucho más focalizada en los oasis ${ }^{26}$, especialmente Dajla ${ }^{27}$ y Jarga, y las rutas a través del desierto. Los oasis mantuvieron una administración estable, con una población permanente a cargo de un funcionario egipcio, aunque se he debatido mucho acerca del grado de autonomía de estas zona, ya que se han encontrado inscripciones en las que el funcionario del oasis "usurpa" atributos iconográficos reservados al faraón.

Las prospecciones del Desert Road Survey del Oriental Institute de Chicago $^{28}$ han sacado a la luz inscripciones relacionadas con la reunificación del país tras el Primer Periodo Intermedio, y parece que los ejércitos de Tebas 
del rey Ahmosis emplearon estas rutas para flanquear a sus rivales del norte. Asimismo, se han encontrado inscripciones del reinado de Amenemhat III, probablemente asociadas a patrullas e inspecciones.

\section{CONCLUSIONES}

La primera conclusión que se puede extraer tras este breve análisis, es que la explotación de las zonas fuera del Valle del Nilo durante la dinastía XII se cuenta entre las más intensas de la historia faraónica. Esto tiene que ver con varios factores; en primer lugar, una estabilidad política interna que permite a los faraones enviar grandes expediciones a cargo de funcionarios de confianza. En segudo lugar, una estructura administrativa fuerte capaz de desarrollar estas expediciones de manera eficiente. Y tercero, una situación exterior de relativa tranquilidad, que permite garantizar hasta cierto punto la seguridad de estas expediciones.

$\mathrm{Y}$ es que esta actividad expedicionaria se empieza a desarrollar de forma intensa en el reinado de Senusert I, faraón que consigue someter de manera firme a la población de la Baja Nubia. Su predecesor, Amenemhat I, lo intentó, pero parece que sus esfuerzos fueron insuficientes. La escasez de expediciones en su reinado se debe fundamentalmente a la profunda remodelación a la que somete al país, trasladando la capital de Tebas a Ity-Tauy en el Delta. Un caso similar sucede en el reinado de Senusert III. Este faraón, famoso por sus campañas militares (que llevarían a su divinización en la Baja Nubia hasta tiempos muy posteriores), apenas dejó testimonios de expediciones comerciales.

Como contraste, véase el caso de Senusert I o Amenemhat III, faraones de reinados largos que reactivaron de forma significativa la explotación de estos recursos, percibiéndose en ellos, especialmente en Amenemhat III, un mayor interés por la diplomacia que por la guerra. Este clima de paz es el que permitió el desarrollo de lo que es el Reino Medio para la egiptología: la edad de oro de la cultura faraónica.

\section{NOTAS}

*Beneficiario de una Beca de Formación del Personal Universitario (FPU) de la Universidad de Salamanca.

${ }^{1}$ Sobre el periodo ver, por ejemplo, OBSOMER, C., Sésotris Ier. Etude chronologique et historique du règne, Bruselas, 1995 ; TALLET, P., Sésostris III et la fin de la XIIe dynastie, París, 2005 ; FAVRY, N., Sésostris Ier et le début de la XIIe dynastie, París, 2009.

${ }^{2}$ O'CONNOR, D., Ancient Nubia: Egypt's Rival in Africa, Filadelfia, 1993, p. 22. 
${ }^{3}$ Sobre la ciudad de Kerma, ver BONNET, Ch., Le temple principal de la ville de Kerma et son quartier religieux: Mission archéologique de l'université de Genève à Kerma, Génova, 2004 y BONNET, Ch., Edifices et rites funéraire à Kerma, Génova, 2000.

${ }^{4}$ Los catálogos documentales más importantes sobre la Baja Nubia son ŽABA, Z., The Rock Inscriptions of Lower Nubia, Praga, 1974 y HINTZE, F., REINEKE, W. F., HINTZE, U. y BURKHARDT, A., Felseninschriften aus dem sudanesischen Nubien, Berlin, 1989.

${ }^{5}$ Sobre la áreas de explotación aurífera, ver KLEMM, D. D., KLEMM, R. M., y MURR, A., «Ancient Gold Mining in the Eastern Desert of Egypt and the Nubian Desert of Sudan», en FRIEDMAN, R. (Ed.), Egypt and Nubia: Gifts of the Desert, Londres, 2002, pp. 215-231.

${ }^{6}$ Sobre las fortalezas y la ocupación egipcia de la Baja Nubia, ver OBSOMER, C., Sésotris Ier. Etude chronologique et historique du règne, Bruselas, 1995, pp. 233-310.

${ }^{7}$ Una descripción de Uadi el-Hudi y sus restos arqueológicos puede encontrarse en SHAW, I. y JAMESON, R.: «Amethyst mining in the Eastern Desert: A Preliminary Survey at Wadi el-Hudi», JEA 79 (1993), pp. 81-97.

${ }^{8}$ FAKHRY, A.: The Inscriptions of the Amethyst Quarries at Wadi El Hudi, El Cairo, 1952; SADEK, A. I.: The Amethyst Mining Inscriptions of Wadi el-Hudi. 2 vols., Warminster, 1980; SEYFRIED, K. J.: Beiträge zu den Expeditionen des Mittleren Reiches in die Ost-Wüste, Hildesheim, 1981.

${ }^{9}$ Sobre la terminología egipcia en relación a los pueblos limítrofes, ver LORTON, D., «The So-called "Vile" Enemies of the King of Egypt (in the Middle Kingdom and Dyn. XVIII)», JARCE 10 (1973), pp. 65-70.

${ }^{10}$ Sobre el yacimiento arqueológico, ver ABD EL-RAZIQ, M.; CASTEL, G.; TALLET, P. y GHICA, V., Les Inscriptions d'Ayn Soukhna, (MIFAO 122), El Cairo, 2002.

${ }^{11}$ Ayn Sujna no 6, ABD EL-RAZIQ et al., op. cit., pp. 108-113.

${ }^{12}$ CASTEL, G. y SOUKKIASSIAN, G., Gebel el-Zeit I: Les mines de galène (Egypte, IIe millénaire av. J.-C.), 1989, y REGEN, I. y SOUKIASSIAN, G., Gebel el-Zeit II: Le materiel inscrit, El Cairo, 2008.

${ }^{13}$ SAYED, A. M. A. H., «Discovery of the Site of the 12th Dynasty Port at Wadi Gawasis on the Red Sea Shore», Revue d'Egyptologie 29 (1977), pp. 140-178.

${ }^{14}$ DIEGO ESPINEL, A., «Los contactos comerciales entre Egipto y Punt durante el Reino Medio», BAEDE 13 (2003), pp. 67-108; DIEGO ESPINEL, A., Abriendo los caminos de Punt. Contactos entre Egipto y el ámbito afro-árabe durante la Edad del Bronce (c. 3000-1065 a. C.), Barcelona , 2009.

${ }^{15}$ KLEMM, R. y KLEMM, D. D., Stones and Quarries in Ancient Egypt, Londres, 2008, pp. 297-311.

${ }^{16}$ COUYAT, J. y MONTET, P., Les inscriptions hiéroglyphiques et hiératiques du Wadi Hammamat, (MIFAO 34), 2 vols., El Cairo, 1912.

${ }^{17}$ Ibídem, pp. 41-42.

${ }^{18}$ SIDEBOTHAM, S. E., HENSE, M. y NOUWENS, H. M., The Red Land. The Illustrated Archaeology of Egypt's Eastern Desert, El Cairo, 2008, pp. 221-222.

${ }^{19}$ TALLET, P., «Notes sur le Ouadi Maghara et sa région au Moyen Empire», BIFAO 102 (2002), pp. 371-387 ; CHARTIER-RAYMOND, M., «Notes sur Maghara (Sinai)», CRIPEL 10 (1988), pp. 13-22 ; CHARTIER-RAYMOND, M.; GRATIEN, B.; TRAUNECKER, C. 
y VINÇON, J. M., «Les sites miniers pharaoniques du Sud-Sinaï. Quelques notes et observations de terrain», CRIPEL 16 (1994), pp. 31-77.

${ }^{20}$ VALBELLE, D. y BONNET, Ch., Le Sanctuaire d'Hathor, maîtresse de la turquoise. Sérabit elKhadim au Moyen Empire, París, 1996.

${ }^{21}$ BRIQUEL-CHATONNET, F., «Les inscriptions proto-sinaïtiques», en VALBELLE, D. y BONNET, Ch. (eds.), Le Sinaï durant l'Antiquité et le Moyen Age. 4000 ans d'bistorie pur un désert, Paris, 1998, pp. 56-60 ; SASS, B., «Notes on some Proto-Sinaitic and Egyptian Inscriptions from Sinai», en ISRAELIT-GROLL, S. (ed.), Egyptological Studies, Jerusalén, 1982, pp. 360-370; SATZINGER, H., «Syllabic and Alphabetic Script, or the Egyptian Origin of the Alphabet», Aegyptus 82 (2002), pp. 15-26.

${ }^{22}$ BAINES, J., «The Stela of Khusobek: Private and Royal Military Narrative and Values», en OSING, J. y DREYER, G., Form und Mass. Beiträge zur Literatur, Sprache und Kunst des alten Ägypten. Festschrift für Gerhard Fecht, Wiesbaden, pp. 43-61.

${ }^{23}$ Sobre este tema, ver SARETTA, P., Egyptian Perceptions of West Semites in Art and Literature During the Middle Kingdom (An Archaeological, Art Historical and Textual Survey), Tesis Doctoral, UMI, Nueva York, 1997.

${ }^{24}$ Malek, J. y Quirke, S., «Memphis, 1991 : Epigraphy», JEA 78 (1992), pp. 13-18.

${ }^{25}$ ALLEN, J. P., «The Historical Inscription of Khnumhetep at Dahshur: Preliminary Report), BASOR 352 (2008), pp. 29-39.

${ }^{26}$ GIDDY, L., Egyptian Oases, Warminster, 1986.

${ }^{27}$ BAUD, M., COLIN F. y TALLET, P., « Les gouverneurs de l'oasis de Dakhla au Moyen Empire», BIFAO 98 (1998), pp. 1-19.

${ }^{28}$ DARNELL, J. C., Theban Desert Road Survey in the Egyptian Western Desert. Volume 1: Gebel Tjauti Rock Inscriptions $1-45$ and Wadi el-Hôl Rock Inscriptions 1-45, (OIP 119), Chicago, 2002. 